\title{
THE NEED FOR HIGH RESOLUTION FOR POLARIZATION STUDIES OF GALACTIC BACKGROUND RADIATION
}

\author{
G.L. VERSCHUUR \\ 4802 Brookstone Terr, Bowie, MD 20715 \\ T.A.Th. SPOELSTRA \\ Netherlands Foundation for Research in Astronomy, Dwingeloo
}

\begin{abstract}
Polarization data at 390 and $826 \mathrm{MHz}$ were obtained with the $300-$ foot telescope in February 1987. A survey of selected regions of sky planned for December 1988 had to be postponed. However, our limited data at $390 \mathrm{MHz}$ show that the $30^{\prime}$ beam detected polarization temperatures between four to six times larger than found in surveys with a 1.3 arcmin resolution. This was true in both the highly polarized region around $1=140$ degrees and in the North Polar Spur where polarization structures appear to be unresolved $(<0.9 \mathrm{pc}$ at the distance of the spur). High resolution observations will be critical to our understanding of the interstellar magnetic field and the scale-length of depolarizing structures.
\end{abstract}

This research was carried out with the aid of a NATO Collaborative Research Grant. 